\title{
Schwinger effect in compact space
}

\author{
Prasant Samantray ${ }^{1, *}$ and Suprit Singh ${ }^{2, \dagger}$ \\ ${ }^{1}$ Department of Physics, BITS-Pilani Hyderabad Campus, Jawahar Nagar, \\ Shamirpet Mandal, Secunderabad 500078, India \\ ${ }^{2}$ Department of Physics, Indian Institute of Technology Delhi, New Delhi 110 016, India
}

(Received 23 March 2021; accepted 25 May 2021; published 16 June 2021)

\begin{abstract}
We consider a theory of scalar QED on a spatially compact $1+1$-dimensional spacetime. By considering a constant electric field pointing down the compact dimension, we compute the quantum effective action by integrating out the scalar degrees of freedom in the Euclidean sector. Working in the saddle-point approximation, we uncover two novel branches/physical regimes upon analytically continuing back to real time and discover a new result, hitherto unreported in previous literature. Implications of our results are discussed.
\end{abstract}

DOI: 10.1103/PhysRevD.103.125012

\section{BACKGROUND}

It is a well-known result of quantum field theory in external backgrounds that strong fields can lead to particle creation from vacuum. The first such prediction was in quantum electrodynamics - that of pair creation by strong electric fields-computed by Schwinger in 1951 [1,2]. In the almost 70 years that have passed, this effect still awaits confirmation in laboratories amidst ongoing efforts. This is in part due to the very nature of the phenomenon that even the leading-order term is exponentially suppressed in flat spacetime. For an excellent general overview the reader is encouraged to look up Refs. [3-9] and references therein. Additionally, with the hope of enhancing the effect and other implications, the mechanism has been probed at finite temperatures [10-14], and also in curved spacetimes such as de Sitter and anti-de Sitter backgrounds [15].

We consider the Schwinger effect in 1+1-dimensional flat spacetime with a compact spatial dimension. This idea has been explored before [16,17] but not with the full rigor of computing the effective action and ascertaining particle creation from it. There also appears to be a lot of confusion in the literature, specifically in the context of compact spatial dimensions or in the presence of a thermal bath when considering particle production via the Schwinger process. With the aim of demystifying this issue, we work this out for scalar quantum electrodynamics, and in doing

*prasant.samantray@ hyderabad.bits-pilani.ac.in suprit@iitd.ac.in

Published by the American Physical Society under the terms of the Creative Commons Attribution 4.0 International license. Further distribution of this work must maintain attribution to the author(s) and the published article's title, journal citation, and DOI. Funded by SCOAP ${ }^{3}$. so obtain a new result which has been absent in the previous analyses. Finally, to answer the question as to why one should consider the effect in a compact dimension, the extra (large) dimensions are often invoked in, for instance, braneworld models (see Refs. [18-20] for string-inspired models) and it is necessary we devise tests to probe these extra dimensions. With this motivation, we consider in this article a toy $1+1$-dimensional model which is analytically tractable and will be the basis for future explorations.

\section{SCALAR QED IN 1 + 1 FLAT SPACETIME WITH COMPACT SPATIAL DIMENSION}

Consider a $(1+1)$-dimensional Minkowski space $d s^{2}=$ $-d t^{2}+d x_{1}^{2}$ with compact spatial dimension under the identification $x_{1} \sim x_{1}+L$. The action for a complex scalar field coupled to an electromagnetic field reads

$$
S_{\phi}=-\int \phi^{*}\left[-D_{\mu} D^{\mu}+m^{2}\right] \phi d t d x_{1},
$$

where $D_{\mu}=\partial_{\mu}-i q A_{\mu}$. We shall work in Euclidean time, $x_{0}=i t$ such that $d s_{E}^{2}=\delta_{a b} d x^{a} d x^{b}=d x_{0}^{2}+d x_{1}^{2}$ and $\quad F_{t x}=\partial_{t} A_{x_{1}}-\partial_{x_{1}} A_{t} \rightarrow i\left(\partial_{x_{0}} A_{x_{1}}-\partial_{x_{1}} A_{x_{0}}\right)=i F_{x_{0} x_{1}}$. The Euclidean action (labeled by the subscript $E$ ) is then given by

$$
\begin{aligned}
S^{E}= & \int d x_{0} d x_{1}\left[\phi^{*}\left(-\nabla^{2}+m^{2}\right) \phi+2 i q \phi^{*} \mathbf{A} \cdot \nabla \phi\right. \\
& \left.+i q|\phi|^{2} \nabla \cdot \mathbf{A}+q^{2}|\phi|^{2} A^{2}\right],
\end{aligned}
$$

with the definition $\nabla \equiv\left(\partial_{x_{0}}, \partial_{x 1}\right)$. We now make the gauge choice $A_{x_{0}}=0, A_{x_{1}}=E x_{0}+a$ respecting the periodic boundary conditions where $a \in R$ cannot be gauged away to zero by any gauge transformation (which respects 
periodic boundary conditions). Together these conditions imply the Euclidean version of Lorenz gauge, that is, $\nabla \cdot \mathbf{A}=0$. With this choice, the action becomes

$$
\begin{aligned}
S^{E}= & \int d x_{0} d x_{1}\left[\phi^{*}\left(-\nabla^{2}+m^{2}\right) \phi\right. \\
& \left.+2 i q \phi^{*}\left(E x_{0}+a\right) \partial_{x_{1}} \phi+q^{2}|\phi|^{2}\left(E x_{0}+a\right)^{2}\right] .
\end{aligned}
$$

In order to compute the effective action, it is a standard procedure to integrate out the scalar field in the path integral, which formally yields

$$
S_{\text {eff }}^{E}=\ln \operatorname{det} \hat{D}=\operatorname{Tr} \ln \hat{D}=\int_{0}^{\infty} \frac{d s}{s} \operatorname{Tr}\left(e^{-s \hat{D}}\right),
$$

where the operator $\hat{D}$ can be read off from the action of the scalar field as

$\hat{D}=-\nabla^{2}+m^{2}+2 i q\left(E x_{0}+a\right) \partial_{x_{1}}+q^{2}\left(E x_{0}+a\right)^{2}$.

Since the differential operator $\hat{D}$ is invariant under translations in the spatial dimension and given the fact that the eigenfunctions of $\hat{D}$ need to obey the periodic boundary conditions in the coordinate $x_{1}$, we can factorize the eigenfunctions (with proper normalization) as

$$
\frac{1}{\sqrt{L}} f_{n}\left(x_{0}\right) \exp \left(-\frac{2 \pi i n x_{1}}{L}\right) \quad \forall n \in \mathbb{Z} .
$$

This yields

$$
\left[-\frac{\partial^{2}}{\partial x_{0}^{2}}+q^{2} E^{2}\left(x_{0}+\frac{2 \pi n}{q E L}+\frac{a}{E}\right)^{2}+m^{2}\right] f_{n}\left(x_{0}\right)=\lambda f_{n}\left(x_{0}\right),
$$

and defining $y:=\left(x_{0}+2 \pi n / q e L+a / E\right)$ puts it in the more familiar form

$$
\left[-\frac{\partial^{2}}{\partial y^{2}}+q^{2} E^{2} y^{2}\right] g_{n}(y)=\left(\lambda-m^{2}\right) g_{n}(y),
$$

which is that of a quantum harmonic oscillator, and since $-\infty<x_{0}<\infty$ we have $-\infty<y<\infty$ for any fixed $n \in \mathbb{Z}$. By identifying $\omega=2 q E$ as the frequency of the oscillator, the solutions to this equation can therefore be written straightaway in terms of the wave functions of a quantum harmonic oscillator as

$$
\frac{1}{\sqrt{L}} \Psi_{n j}\left(x_{0}+\frac{2 \pi n}{q E L}+\frac{a}{E}\right) \exp \left(-\frac{2 \pi i n x_{1}}{L}\right)
$$

with eigenvalues $\lambda_{j, n}=m^{2}+(2 j+1) q E$, where $j=$ $0,1,2, \ldots \forall n \in \mathbb{Z}$. Since $\lambda_{j, n}$ are explicitly independent of $n$, the eigenfunctions are infinitely degenerate. The heat kernel for the operator $\hat{D}$ can therefore be written as

$$
\begin{aligned}
& K\left(s ; x_{0}^{\prime}, x_{1}^{\prime} \mid x_{0}, x_{1}\right) \\
& =\frac{1}{L} \sum_{n=-\infty}^{\infty} \sum_{j=0}^{\infty} e^{-s \lambda_{j, n}} \Psi_{n, j}^{*}\left(y^{\prime}\right) \Psi_{n, j}^{*}(y) \\
& \times \exp \left(-\frac{2 \pi i n}{L}\left(x_{1}-x_{1}^{\prime}\right)\right) \\
& =\sum_{n=-\infty}^{\infty} k_{n}\left(s ; x_{0}^{\prime} \mid x_{0}\right) \exp \left(-\frac{2 \pi i n}{L}\left(x_{1}-x_{1}^{\prime}\right)\right) \text {, }
\end{aligned}
$$

where $k_{n}$ is the propagator for the quantum harmonic oscillator for a fixed $n$ in Euclidean time. Since the computation of the effective action involves taking a trace, we can set $x_{0}^{\prime}=x_{0}$ and $x_{1}^{\prime}=x_{1}$ in the heat kernel, which gives

$k_{n}\left(s ; x_{0} \mid x_{0}\right)=\frac{1}{L} e^{-m^{2} s}\left(\frac{q E}{2 \pi \sinh (2 q E s)}\right)^{\frac{1}{2}} e^{-q E \tanh (q E s) y^{2}}$,

and the complete heart kernel (in the coincidence limit) is given by

$$
\begin{aligned}
K\left(s ; x_{0}, x_{1} \mid x_{0}, x_{1}\right)= & \frac{1}{L} e^{-m^{2} s}\left(\frac{q E}{2 \pi \sinh (2 q E s)}\right)^{\frac{1}{2}} \\
& \times \sum_{n=-\infty}^{\infty} e^{-q E \tanh (q E s) y^{2}},
\end{aligned}
$$

where $y=\left(x_{0}+2 \pi n / q e L+a / E\right)$ as defined earlier. Using the Poisson summation formula, this can be manipulated to give

$$
\begin{aligned}
& K\left(s ; x_{0}, x_{1} \mid x_{0}, x_{1}\right) \\
& =\frac{q E}{4 \pi} \frac{e^{-m^{2} s}}{\sinh (q E s)} \\
& \quad \times \sum_{n=-\infty}^{\infty} \exp \left(-\frac{n^{2} L^{2} q E \operatorname{coth}(q E s)}{4}-i q n\left(E x_{0}+a\right) L\right) .
\end{aligned}
$$

With this, we can write the Euclidean effective action as

$$
\begin{aligned}
S_{\mathrm{eff}}^{E} & =-\int_{0}^{\infty} \frac{d s}{s} \operatorname{Tr}\left(e^{-s \hat{D}}\right) \\
& =-\int d x_{0} d x_{1} \int_{0}^{\infty} \frac{d s}{s} K\left(s ; x_{0}, x_{1} \mid x_{0}, x_{1}\right) \\
& =\int d x_{0} d x_{1} L_{\mathrm{eff}}^{E}
\end{aligned}
$$

from which we can read off the effective Lagrangian 


$$
\begin{aligned}
L_{\text {eff }}^{E}= & -\sum_{n=-\infty}^{\infty} e^{-i q n L\left(E x_{0}+a\right)} \int_{0}^{\infty} \frac{d s}{4 \pi s^{2}} e^{-m^{2} s} \frac{q E s}{\sinh q E s} \\
& \times \exp \left(-\frac{n^{2} L^{2} q E \operatorname{coth}(q E s)}{4}\right) .
\end{aligned}
$$

Interestingly, the prefactor before the integral is actually $\exp (-i q \oint \mathbf{A} \cdot \mathbf{d x})$ and corresponds to the geometric phase. This geometric phase from its very form is gauge invariant and is the result of the fact that the Euclidean topology considered here is not simply connected. This nontrivial holonomy results in the prefactor as above. A similar prefactor appears even in computations of the Casimir energy in compact spacetimes, where the integer $n$ takes specific values, leading to the minimization of the effective potential that gives the vacuum energy density of the spacetime (details can be found in Ref. [21]). This term is usually not reported in the literature. We shall drop this term for the calculations in this article in order to focus on the pair-production rate. With a change of variables $\tau=$ $q E s$ and defining the parameters $\lambda_{E}=m^{2} / q E, R_{E}=$ $m / q E$ and $\sigma_{E}=L / 2 R_{E}$ (where now the subscripts indicate Euclidean time) and splitting the $n=0$ and $n \neq 0$ terms, we have

$$
\begin{aligned}
L_{\mathrm{eff}}^{E}= & -\frac{q E}{4 \pi} \int_{0}^{\infty} \frac{d \tau}{\tau \sinh \tau} e^{-\lambda_{E} \tau} \\
& -\frac{q E}{2 \pi} \sum_{n=1}^{\infty} \int_{0}^{\infty} \frac{d \tau}{\tau \sinh \tau} e^{-\lambda_{E}\left(\tau+n^{2} \sigma_{E}^{2} \operatorname{coth} \tau\right)} \\
= & L_{\mathrm{eff}}^{E(0)}+L_{\mathrm{eff}}^{E(n>0)} .
\end{aligned}
$$

This will be analytically continued back to real time with $t=-i x_{0}, E_{\text {phy }}=i E, \sigma=i \sigma_{E}$, and $\lambda=-i \lambda_{E}$, where the latter two imply $\sigma_{E} \lambda_{E}=\sigma \lambda$. Considering the first term (which is also independent of $\sigma_{E}$ ) after analytically continuing it back to real time,

$$
L_{\mathrm{eff}}^{(0)}=\frac{q E_{\mathrm{phy}}}{4 \pi} \int_{0}^{\infty} \frac{d \tau}{\tau \sin \tau} e^{-\lambda \tau},
$$

we see that there exist simple poles at $\tau=k \pi$ with $k=1,2, \ldots$ The pole at $\tau=0$ does not contribute as it is canceled by the background shift in the limit of vanishing electric field. Therefore, to evaluate the integral we can express $\tau=k \pi+\epsilon \exp (i \theta)$ in the complex $\tau$ plane. The pole at $\tau=0$ does not contribute to the imaginary part as it is canceled by the background in the limit that the electric field goes to zero. The contour individually circles each of the poles that lie on the real axis, which contribute to give

$$
L_{\text {eff }}^{(0)}=\frac{i q E_{\text {phy }}}{4 \pi} \sum_{k=1}^{\infty} \frac{(-1)^{k+1}}{k} e^{-\lambda k \pi} .
$$

This is the standard expression for the particle-production rate in flat $1+1$-dimensional spacetime without any compactification. As for the second term, we can use the saddle-point approximation (only in the limit where $\left.\lambda_{E} \gg 1\right)$ to evaluate this Laplace-type integral, which is of the form

$$
I=\int_{a}^{b} g(\tau) e^{-\lambda_{E} f(\tau)} \simeq \sqrt{\frac{2 \pi}{\lambda_{E} f^{\prime \prime}\left(\tau_{0}\right)}} g\left(\tau_{0}\right) e^{-\lambda_{E} f\left(\tau_{0}\right)},
$$

with $g(\tau)=1 / \tau \sinh \tau$ and $f(\tau)=\tau+n^{2} \sigma_{E}^{2} \operatorname{coth} \tau$. Noting that

$$
f^{\prime}(\tau)=1-\frac{n^{2} \sigma_{E}^{2}}{\sinh ^{2}(\tau)} ; \quad f^{\prime \prime}(\tau)=\frac{2 n^{2} \sigma_{E}^{2} \cosh \tau}{\sinh ^{3}(\tau)},
$$

the saddle points from the zeros of the derivative $f^{\prime}\left(\tau_{0}\right)=0$ are given by $\sinh \tau_{0}=n \sigma_{E}$, and hence $e^{\tau_{0}}=n \sigma_{E}+$ $\sqrt{1+n^{2} \sigma_{E}^{2}}$ for $n=1,2,3, \ldots$ Equivalently, we could have solved for the saddle points explicitly as $\tau_{0}=\log \left(n \sigma_{E}+\sqrt{1+n^{2} \sigma_{E}^{2}}\right)$. Therefore, the effective Lagrangian, by summing over the contributions from all of the saddle points, is

$$
\begin{aligned}
L_{\mathrm{eff}}^{E(n>0)} & \simeq-\frac{q E}{2 \pi} \sum_{n=1}^{\infty} \sqrt{\frac{2 \pi}{\lambda_{E} f^{\prime \prime}\left(\tau_{0}\right)}} g\left(\tau_{0}\right) e^{-\lambda_{E} f\left(\tau_{0}\right)} \\
& =-\frac{q E}{\sqrt{4 \pi \lambda_{E}}} \sum_{n=1}^{\infty} \frac{\left.e^{-\lambda_{E}\left[n \sigma_{E}\right.} \sqrt{1+n^{2} \sigma_{E}^{2}}+\sinh ^{-1} n \sigma_{E}\right]}{\sqrt{n \sigma_{E}} \sinh ^{-1} n \sigma_{E}\left(1+n^{2} \sigma_{E}^{2}\right)^{1 / 4}},
\end{aligned}
$$

which will be our master formula. Modulo the choice of gauge (in accordance with the nontrivial holonomy), it is evident that this formula is mathematically unambiguous and therefore unique in 1+1-dimensional compact space. We now have two cases of physical interest to consider.

\section{A. Case: $\sigma>1$ or $L>2 R_{0}$}

The correct analytic continuations are

$$
\begin{gathered}
\sqrt{1+n^{2} \sigma_{E}^{2}} \rightarrow-i \sqrt{n^{2} \sigma^{2}-1}, \\
\sinh ^{-1}(i n \sigma)=i \pi / 2-\cosh ^{-1} n \sigma .
\end{gathered}
$$

We chose these analytic continuations due to the following reasons. Consider a complex multivalued function $f(z)=$ $\log \left(z+\sqrt{1+z^{2}}\right)$. In order to make this function analytic, we choose a branch cut starting at $z=-i$ and running all the way upwards along the imaginary axis to $z=i \infty$ in the complex $z$ plane. This choice of cut ensures that $f(z) \in \mathbb{R}$ for $z \in \mathbb{R}$. Plugging this into the master formula, we get 


$$
\begin{aligned}
L_{\mathrm{eff}}^{(n>0)}= & \frac{i q E_{\mathrm{phy}}}{\sqrt{4 \pi \lambda \sigma}} \\
& \times \sum_{n=1}^{\infty} \frac{e^{-i \lambda\left[-n \sigma \sqrt{n^{2} \sigma^{2}-1}-i \pi / 2+\cosh ^{-1} n \sigma\right]}}{\sqrt{n}\left(-i \pi / 2+\cosh ^{-1} n \sigma\right) e^{-i \pi / 4}\left(n^{2} \sigma^{2}-1\right)^{1 / 4}} .
\end{aligned}
$$

Setting $n \sigma=\cosh \left(\alpha_{n}\right)$, since $\sigma>1$ and $n=1,2, \ldots$, we have

$L_{\mathrm{eff}}^{(n>0)}=\frac{i q E_{\mathrm{phy}}}{\sqrt{4 \pi \lambda \sigma}} e^{i \pi / 4} \sum_{n=1}^{\infty} \frac{e^{-i \lambda \alpha_{n}+i(\lambda / 2) \sinh \left(2 \alpha_{n}\right)-\lambda \pi / 2}}{\sqrt{n \sinh \alpha_{n}}\left(-i \pi / 2+\alpha_{n}\right)}$.

This can further be split (noting that $\lambda \sigma=m L / 2$ ) as

$$
\begin{aligned}
L_{\text {eff }}^{(n>0)}= & \frac{i q E_{\text {phy }}}{\sqrt{2 \pi m L}} e^{-\lambda \pi / 2} \sum_{n=1}^{\infty} \frac{\left[(\pi / 2) \sin \gamma_{n}+\alpha_{n} \cos \gamma_{n}\right]}{\sqrt{n \sinh \alpha_{n}}\left(\pi^{2} / 4+\alpha_{n}^{2}\right)} \\
& +\operatorname{Re}\left(L_{\text {eff }}\right),
\end{aligned}
$$

where $\gamma_{n}=\lambda \alpha_{n}-(\lambda / 2) \sinh \left(2 \alpha_{n}\right)-\pi / 4$. The complete effective Lagrangian for $\sigma>1$ is

$$
\begin{aligned}
L_{\mathrm{eff}}= & \frac{i q E_{\mathrm{phy}}}{4 \pi} \sum_{n=1}^{\infty} \frac{(-1)^{n+1}}{n} e^{-\lambda n \pi}+\frac{i q E_{\mathrm{phy}}}{\sqrt{2 \pi m L}} e^{-\lambda \pi / 2} \\
& \times \sum_{n=1}^{\infty} \frac{\left[(\pi / 2) \sin \gamma_{n}+\alpha_{n} \cos \gamma_{n}\right]}{\sqrt{n \sinh \alpha_{n}}\left(\pi^{2} / 4+\alpha_{n}^{2}\right)}+\operatorname{Re}\left(L_{\mathrm{eff}}\right) .
\end{aligned}
$$

Further, for $\sigma \gg 1$, only the $n=1$ order term contributes maximally, so that

$$
\begin{aligned}
L_{\text {eff }} \approx & \frac{i q E_{\text {phy }}}{4 \pi} e^{-\lambda \pi}+\frac{i q E_{\text {phy }}}{\sqrt{2 \pi m L}} e^{-\lambda \pi / 2} \frac{1}{\sqrt{\sigma} \ln \sigma} \\
& +\operatorname{Re}\left(L_{\text {eff }}\right) .
\end{aligned}
$$

The vacuum-to-vacuum expectation value is

$$
\langle\text { out }| \text { in }\rangle=\exp \left[i S_{\text {eff }}\right]=\exp \left[i L T L_{\text {eff }}\right],
$$

where $L$ is the volume of the compact space and $T$ is the time interval under consideration. The pair-production probability, which is $\mathbb{P}=1-\mid\langle$ out $|$ in $\rangle\left.\right|^{2}$, picks up only the imaginary part of the effective Lagrangian, giving

$$
\mathbb{P} \approx L T\left[\frac{q E_{\text {phy }}}{2 \pi} e^{-\lambda \pi}+\frac{2 q E_{\text {phy }}}{\sqrt{2 \pi m L \sigma} \ln \sigma} e^{-\lambda \pi / 2}\right] .
$$

This is an entirely new result valid for $\sigma \gg 1$ (along with $\lambda \sigma \gg 1$ ), that is, in the case of a large compact dimension, the $\sigma$-dependent term appears to enhance the standard Schwinger pair-creation rate. Further, as a consistency check, in the limit $\sigma \rightarrow \infty$ it reduces to the standard expression for the Schwinger effect in $1+1$ Minkowski spacetime with flat topology.

\section{B. Case: $\sigma<1$ or $L<2 R_{0}$}

In this case the correct analytic continuations are

$$
\begin{aligned}
& \sqrt{1+n^{2} \sigma_{E}^{2}} \rightarrow \sqrt{1-n^{2} \sigma^{2}}, \\
& -\sinh (i n \sigma)=\sinh \left(n \sigma_{E}\right) \rightarrow-i\left[\sin ^{-1} n \sigma+2 k \pi\right],
\end{aligned}
$$

with $k=0,1,2, \ldots$ for $n \sigma<1$ with $n=1,2,3, \ldots,[1 / \sigma]$, where $[x]$ is the greatest integer function. Plugging this into the master formula, we get

$$
\begin{aligned}
L_{\mathrm{eff}}^{(n>0)}= & \frac{q E_{\text {phy }}}{\sqrt{4 \pi \lambda}} \\
& \times \sum_{k=1}^{\infty} \sum_{n=1}^{n_{\max }} \frac{e^{-\lambda\left[n \sigma \sqrt{1+n^{2} \sigma^{2}}+\sin ^{-1} n \sigma+2 \pi k\right]}}{\sqrt{n \sigma}\left[\sin ^{-1}(n \sigma)+2 \pi k\right]\left(1+n^{2} \sigma^{2}\right)^{1 / 4}} .
\end{aligned}
$$

Therefore, the total effective Lagrangian is

$$
\begin{aligned}
L_{\mathrm{eff}}= & i \frac{q E_{\mathrm{phy}}}{4 \pi} \sum_{n=1}^{\infty} \frac{(-1)^{n+1}}{n} e^{-\lambda n \pi} \\
& +\frac{q E}{\sqrt{4 \pi \lambda}} \sum_{k=1}^{\infty} \sum_{n=1}^{n_{\max }} \frac{e^{-\lambda\left[n \sigma \sqrt{1+n^{2} \sigma^{2}}+\sin ^{-1} n \sigma+2 \pi k\right]}}{\sqrt{n \sigma}\left[\sin ^{-1}(n \sigma)+2 \pi k\right]\left(1+n^{2} \sigma^{2}\right)^{1 / 4}},
\end{aligned}
$$

where $n_{\max }=[1 / \sigma]$. It is noteworthy that only the first term contributes to the imaginary part of the effective action, giving particle production that is independent of $\sigma$ and hence the compactification scale $L$ in this case, and the Schwinger mechanism is identical to that in normal flat spacetime. The second term is real and therefore goes into correcting the Maxwell action in 1 + 1-dimensional spacetime. Also, the $n=1$ lowest-order term coincides with results discussed in Refs. [16,22] in the context of flux discharge and decay of the electric field.

\section{DISCUSSION}

The Schwinger effect - that is, particle production due to vacuum decay induced by external fields-is still one of the outstanding radical predictions of quantum electrodynamics and quantum field theory in external backgrounds in general. In fact, the mechanism in its various appearances is also a basis for various theoretical strides in gravitation and cosmology, and even theoretical condensed matter physics. The inverse exponential dependence on an external electric field suppresses the mechanism requiring strong fields. A way forward is to seek arenas where enhancement of the effect can occur with some augmentation over the usual 
Poincaré-invariant flat spacetime. We considered the effect in scalar quantum electrodynamics in 1+1-dimensional flat spacetime with a compact spatial dimension. The motivation for this is twofold: as a check for indications of enhancement, and as a step towards devising tests for large extra dimensions invoked in string-inspired models.

Through rigorous computations of the effective action, we found results previously unstated in the literature. The calculations were done in the Euclidean sector with zero ambiguities. Remarkably, the Euclidean sector contains information about both regimes of interest, viz., (a) $\sigma<1 \Rightarrow L<2 m / q E$ and (b) $\sigma>1 \Rightarrow L>2 m / q E$ when rotated back to real time. There is a standard term (independent of the compactification scale or " $\sigma$ ") that always contributes to particle production in both cases. For case (a) the compactification has no effect on the rate of particle production. Certain previous studies have reported that there is no particle production in this regime, since in the usual instanton approach the energy density of the electric field does not drop to the requisite amount to create an on-shell real particle pair. In this picture a virtual pair is created at a spatial point and then separates in imaginary time until they meet up at the antipodal point and annihilate. However, this picture of energy conservation is heuristic and possibly incomplete in the context of small compact spatial dimensions with nontrivial holonomy. On the other hand, for case (b) we predicted a new rate obtained via analytic continuation from the Euclidean sector. This can have implications for the early Universe cosmology and braneworld models. We note that there is a discontinuity at $L=2 \mathrm{~m} / q E$ upon analytic continuation back to real time. This can be attributed to the saddle-point approximation, and possibly the situation will be remedied if a complete quantum or numerical analysis is performed. It is important to note that a central tenet of our analysis is that we assumed $\sigma_{E} \lambda_{E}=\sigma \lambda \gg 1$ for both of the regimes. This in turn translates into the simple condition that $m L \gg 1$, which basically implies that the compactification scale is much larger than the Compton wavelength of the particle, thereby allowing a semiclassical analysis to hold. Regimes where $m L \ll 1$ would have to be treated by considering the full effects of backreaction on the gauge field sector as well.

Additionally, our results can be generalized in a straightforward manner even to the Schwinger mechanism at finite temperature but without any spatial compactification. This is possible since from the perspective of the Euclidean sector it is just a relabeling of the coordinate axes and we would have to identify the Euclidean time coordinate $x_{0} \sim x_{0}+\beta$ instead. We can then read off the effective Lagrangian by switching $L \rightarrow \beta$. We rediscovered the temperature regimes $T>T_{c}$ and $T<T_{c}$ reported previously in the literature $[10,16]$, where $T_{c}=1 / R_{0}=q E / 2 m$. The interesting thing to note is that for $T>T_{c}$, there appears to be no effect on the Schwinger process and the $n=0$ term in our master formula remains the relevant rate. This is completely in line with the results of Refs. [13,14]. However, for the regime $T<T_{c}$ our calculations showed that thermality does contribute to the one-loop effective action previously unreported in the literature. This sharp behavior at $\sigma=1$ (for the spatially compact case) and for $T=T_{c}$ (in the thermal case) is likely indicative of either 1) the need to go beyond the one-loop order and beyond the saddle-point approximation while evaluating the master formula, or 2) an intricate structure of the effective action owing to the choice of gauge respecting the periodic boundary conditions (thereby leading to the nontrivial holonomy) and working entirely in the Euclidean formalism. There appears not to be any general consensus on this yet in the present literature. For more details on this contentious issue, we refer to Refs. [23-33].

Our analysis has implications for models of cosmology that have large extra dimensions as a feature. Even though our analysis was done strictly in $1+1$ dimensions, we can nevertheless draw some qualitative inferences. From Eq. (24), we can see that there is an exponential enhancement for the rate of particle production as the compactification scale approaches $\sigma \rightarrow 1$ from above. This indicates that as the large extra dimensions got compactified in the inflationary era via some dynamical mechanism, the Universe was populated with charged particles at an exponentially faster rate compared to the usual Schwinger result without any extra dimensions. This would in turn have resulted in a faster shorting of the electric field at extragalactic scales. With the production of charged particles enhanced via both the inflationary expansion and the mechanism stated in Eq. (24), this would have rapidly increased plasma conductivity in the early inflationary era, thereby influencing the generation and evolution of primordial electromagnetic fields. For astrophysical implications, the reader is urged to look at Ref. [34] and references therein. Additionally, as remarked earlier, since we worked entirely in the Euclidean sector, our result (24) is applicable for finite-temperature systems as well with the identification that $L=1 / T$. Therefore, a more earthly application of our result can be in the context of bilayer graphene [16] with a mass gap of a few hundred $\mathrm{MeV}(\sim 200 \mathrm{MeV})$, a total voltage drop across a sample size (8-10 microns) of the order of around 500-600 MeV, and at a temperature $T \sim 200 \mathrm{~K}$ so that we have $T<T_{c}$. With modern technology, one should in principle be able to test the validity of our result by directly measuring the rate of particle production.

It is important to note that we did not consider backreaction in our analysis and that issue is still outstanding. For one, it is not clear how the imaginary term of the effective action and hence particle production can be accounted for in modifying the classical equations for the background without any artificial manipulations. 
A self-consistent approach to handle backreaction is still pending, possibly through semianalytical and/or numerical techniques. This will also seek to answer the question of the fate of the QED vacuum in compact space by incorporating backreaction as well as inhomogeneous fields on compact spacetimes, including a study in higher than the two dimensions considered in this article.

\section{ACKNOWLEDGMENTS}

The research of P. S. is partially supported by CSIR Grant No. 03(1350)/16/EMR-II Government of India, and also by the OPERA fellowship from BITS-Pilani, Hyderabad Campus. The research of S. S. is partially supported by a Young Faculty Incentive Fellowship from IIT Delhi, India.
[1] W. Heisenberg and H. Euler, Z. Phys. 98, 714 (1936).

[2] J. Schwinger, Phys. Rev. 82, 664 (1951).

[3] J. D. Brown and C. Teitelboim, Nucl. Phys. B297, 787 (1988); J. Garriga, Phys. Rev. D 49, 6327 (1994).

[4] G. V. Dunne, Heisenberg-Euler effective Lagrangians: Basics and extensions, in From Fields to Strings, edited by M. Shifman et al. (2005), Vol. 1, pp. 445-522, https:// doi.org/10.1142/9789812775344_0014.

[5] G. V. Dunne, Int. J. Mod. Phys. A 25, 2373 (2010).

[6] I. K. Affleck and N. S. Manton, Nucl. Phys. B194, 38 (1982).

[7] I. K. Affleck, O. Alvarez, and N. S. Manton, Nucl. Phys. B197, 509 (1982).

[8] K. Srinivasan and T. Padmanabhan, Phys. Rev. D 60, 024007 (1999).

[9] T. Padmanabhan, Eur. Phys. J. C 78, 563 (2018).

[10] L. Medina and M. C. Ogilvie, Phys. Rev. D 95, 056006 (2017).

[11] S. P. Kim and H. K. Lee, Phys. Rev. D 76, 125002 (2007).

[12] S. P. Kim, H. K. Lee, and Y. Yoon, Phys. Rev. D 79, 045024 (2009).

[13] H. Gies, Phys. Rev. D 60, 105002 (1999).

[14] H. Gies, Phys. Rev. D 61, 085021 (2000).

[15] S. P. Kim and D. N. Page, Phys. Rev. D 78, 103517 (2008); R. G. Cai and S. P. Kim, J. High Energy Phys. 09 (2014) 72; W. Fischler, P. H. Nguyen, J. F. Pedraza, and W. Tangarife, Phys. Rev. D 91, 086015 (2015); P. Samantray, J. High Energy Phys. 04 (2016) 060; P. Samantray and S. Singh, Phys. Rev. D 99, 085006 (2019); R. Sharma and S. Singh, Phys. Rev. D 96, 025012 (2017).
[16] A. Brown, Phys. Rev. D 98, 036008 (2018).

[17] Y. Qiu and L. Sorbo, Phys. Rev. D 102, 045010 (2020).

[18] Z. Wu, arXiv:2010.11528v1.

[19] Q. Jia, J. X. Lu, Z. Wu, and X. Zhu, Nucl. Phys. B953, 114947 (2020).

[20] J. X. Lu, Phys. Lett. B 788, 480 (2019).

[21] L. H. Ford, Phys. Rev. D 21, 933 (1980).

[22] M. Kleban, K. Krishnaiyengar, and M. Porrati, J. High Energy Phys. 11 (2011) 096.

[23] P. H. Cox, W. S. Hellman, and A. Yildiz, Ann. Phys. (N.Y.) 154, 211 (1984).

[24] M. Loewe and J. C. Rojas, Phys. Rev. D 46, 2689 (1992).

[25] P. Elmfors and B.-S. Skagerstam, Phys. Lett. B 348, 141 (1995).

[26] A. K. Ganguly, P. K. Kaw, and J. C. Parikh, Phys. Rev. C 51, 2091 (1995).

[27] J. Hallin and P. Liljenberg, Phys. Rev. D 52, 1150 (1995).

[28] O. Gould and A. Rajantie, Phys. Rev. D 96, 076002 (2017).

[29] O. Gould, S. Mangles, A. Rajantie, S. Rose, and C. Xie, Phys. Rev. A 99, 052120 (2019).

[30] O. Gould, A. Rajantie, and C. Xie, Phys. Rev. D 98, 056022 (2018).

[31] P. Draper, Phys. Rev. D 98, 125014 (2018).

[32] G. Torgrimsson, Phys. Rev. D 99, 096007 (2019).

[33] M. Korwar and A. M. Thalapillil, Phys. Rev. D 98, 076016 (2018).

[34] K. Subramanian, Rep. Prog. Phys. 79, 076901 (2016). 